\title{
Die Konfiguration von Wirtschaftssystemen: Ein internationaler Vergleich
}

Vor dem Hintergrund der US-Finanzkrise und der aktuell andauernden Gefahr einer Destabilisierung des Euro-Raums, die durch die „Griechenlandkrise“ ihren Nährboden erhalten hat, drängt sich ein tieferer Blick in die internationalen Wirtschafts- und Finanzsysteme auf. Grundsätzlich charakterisiert man das System eines Landes entweder als bank-basiert, wenn monetäre Finanzintermediäre (Banken), Pensionsfonds und Versicherungen die Volkswirtschaft dominieren, oder als markt-basiert, wenn der Kapitalmarkt hoch entwickelt ist und eine intensive Verflechtung mit der Volkswirtschaft aufweist (Allen/Gale 1995).

\section{Die Rivalität zwischen bank- und marktbasierten Volkswirtschaften}

In der Literatur kategorisiert man Wirtschafts- und Finanzsysteme entweder als bank- oder markt-basierte Systeme, wobei Deutschland (bank-basiert) und Amerika (markt-basiert) als Musterbeispiele gelten. Während in dem ersten System konservative Finanzintermediäre, wie z.B. Banken, die wichtigste Rolle in der Mediation zwischen Investoren und Gläubigern spielen, übernimmt in dem zweiten System der Kapitalmarkt selbst die direkte Vermittlung. Empirische Studien bezüglich der Dominanz eines der beiden Systeme bezogen auf ein nachhaltiges Wachstum des Bruttoinlandsprodukts zeichnen kein eindeutiges Bild. Während Tadesse (2000) in einer 36-Länder-Studie eine Überlegenheit des markt-basierten Systems feststellt, wenn der Finanzsektor weit entwickelt ist, findet Levine (2002) in einer Analyse von 48 Ländern keine Anzeichen für eine grundsätzliche Dominanz eines Systems. Vielmehr präge die Qualität der Institutionen der Volkswirtschaft, insbesondere das Rechtssystem, die wirtschaftliche Entwicklung. Obwohl es keine eindeutigen Belege für die Unterlegenheit des bank-basierten Systems gibt, wurde beginnend in den 1990er Jahren der Niedergang des Bankensektors erwartet (Miller 1997). Eine Studie von Allen/
Santomero (2001) belegt diese Erwartung für die amerikanische Volkswirtschaft, da Banken ihr Geschäftsmodell von klassischen zinsgetriebenen Tätigkeiten, wie z. B. Kreditvergabe an Unternehmen, zu mehr provisionsgetriebenen Instrumenten, wie z.B. die Platzierung von Aktien und Anleihen, ändern. Durch diese Anpassung verliert der Bankensektor seine direkte Beziehung zu Schuldnern oder Gläubigern, da Transaktionstätigkeiten an Bedeutung gewinnen, während z. B. die Überwachung von Schuldnern (Unternehmenskredit) an Anreiz verliert. Schmidt et al. (1999) zeigen in ihrer Analyse auf, dass eine ähnliche Entwicklung wie in den USA in Europa für Deutschland und England nicht festzustellen ist, während Frankreich starke Tendenzen dazu aufweist. Als zentrales Analyseergebnis der Studie stellen die Autoren fest, dass in allen drei europäischen Ländern eine Zunahme der Verflechtungen innerhalb des Finanzsektors zu erkennen ist.

Das Ziel der vorliegenden Analyse ist, ${ }^{1}$ die gewonnen Ergebnisse der beiden letztgenannten Studien aufzunehmen und die Entwicklung der Wirtschaftssysteme und besonders der Finanzsektoren der wichtigsten europäischen Staaten, Deutschland, Frankreich, England, Spanien und Italien, von 1997 bis 2008 zu untersuchen sowie mit den USA zu vergleichen. Im Folgenden wird kurz die Methodik erläutert (Abschnitt 2), bevor die wesentlichen Ergebnisse präsentiert werden (Abschnitt 3) und ein Fazit (Abschnitt 4) gezogen wird.

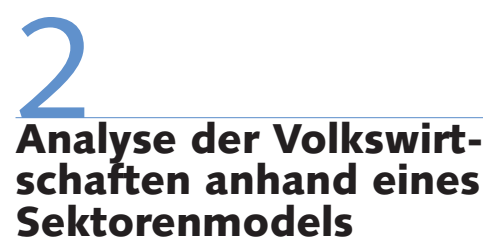

Als Grundlage der Analyse wurden für alle sechs Länder die jeweiligen Zentralbankdaten finanzieller Bestände von Vermögen und Schulden der Volkswirtschaften herangezogen (z.B. Ergebnisse der Gesamtwirtschaftlichen Finanzierungsrechnung in Deutschland). Die jeweiligen Datenbanken sind in Übersicht 1 zusammengefasst.

Für die Analyse werden die Volkswirtschaften in die folgenden sieben Sektoren unterteilt: Haushalte $(\mathrm{HH})$, nicht-finanzielle Unternehmen (NFU), Staat, Rest der Welt (RdW), Banken (MFI), Versicherungen und Pensionsfonds (Versich.) und andere Finanzintermediäre (AFI). Die drei letztgenannten Sektoren können als Finanzsektor zusammengefasst werden,

\footnotetext{
1 Der vorliegende Beitrag illustriert Teile einer empirischen Studie, die beim Autor angefordert werden kann.
}

Florian Kirchner, MSc in General Manage-
ment an der European Business School,
Doktorand der Betriebswirtschaft an der
Johann-Wolfgang-Goethe-Universität
Frankfurt a.M.. Arbeitsschwerpunkte:
Konfiguration von Wirtschaftssystemen und
Finanzierung von Unternehmen.
e-mail: florian.kirchner@ebs.de


Tabelle1: Sektorenmodell der deutschen Wirtschaft zum 31.12.2008, normalisiert

\begin{tabular}{|c|c|c|c|c|c|c|c|c|c|c|c|c|c|c|c|}
\hline & $1 a$ & $1 b$ & $2 a$ & $2 b$ & $3 a$ & $3 b$ & $4 a$ & $4 b$ & $5 a$ & $5 b$ & $6 a$ & $6 b$ & $7 a$ & $7 b$ & \\
\hline Schulden & \multicolumn{2}{|c|}{$\mathrm{HH}$} & \multicolumn{2}{|c|}{ NFU } & \multicolumn{2}{|c|}{ Staat } & \multicolumn{2}{|c|}{$\mathrm{RdW}$} & \multicolumn{2}{|c|}{ MFI } & \multicolumn{2}{|c|}{ Versich. } & \multicolumn{2}{|c|}{ AFI } & Summe \\
\hline Vermögen & verbriefte & $\begin{array}{c}\text { Nicht } \\
\text { verbriefte }\end{array}$ & verbr. & $\begin{array}{c}\mathrm{n} . \\
\text { verbr. }\end{array}$ & verbr. & $\begin{array}{c}\mathrm{n} . \\
\text { verbr. }\end{array}$ & verbr. & $\begin{array}{c}\mathrm{n} . \\
\text { verbr. }\end{array}$ & verbr. & $\begin{array}{c}\mathrm{n} . \\
\text { verbr. }\end{array}$ & verbr. & $\begin{array}{c}\mathrm{n} . \\
\text { verbr. }\end{array}$ & verbr. & $\begin{array}{c}\mathrm{n} . \\
\text { verbr. }\end{array}$ & \\
\hline $\mathrm{HH}$ & - & - & 5 & 17 & 5 & 0 & 13 & 6 & 13 & 86 & 1 & 66 & 20 & 1 & 233 \\
\hline NFU & 0 & 4 & - & - & 1 & 2 & 11 & 15 & 4 & 37 & 3 & 5 & 3 & 0 & 86 \\
\hline Staat & 0 & 1 & 1 & 6 & - & - & 2 & 5 & 1 & 11 & 0 & 0 & 1 & 0 & 30 \\
\hline RdW & 0 & 11 & 14 & 22 & 26 & 3 & - & - & 39 & 61 & 2 & 5 & 4 & 1 & 187 \\
\hline MFI & 0 & 61 & 8 & 68 & 23 & 18 & 26 & 105 & - & - & 1 & 2 & 8 & 6 & 326 \\
\hline Versich. & 0 & 3 & 2 & 9 & 3 & 1 & 8 & 9 & 5 & 29 & - & - & 14 & 0 & 83 \\
\hline AFI & 0 & 1 & 6 & 4 & 7 & 0 & 10 & 3 & 10 & 12 & 1 & 0 & - & - & 54 \\
\hline \multirow[t]{2}{*}{ Summe } & 0 & 81 & 38 & 126 & 66 & 24 & 72 & 143 & 72 & 236 & 7 & 77 & 50 & 8 & 1.000 \\
\hline & & 81 & & 164 & & 89 & & 215 & & 308 & & 84 & & 59 & \\
\hline
\end{tabular}

$\mathrm{HH}=$ Haushalte, $\mathrm{NFU}=$ nicht-finanzielle Unternehmen, $\mathrm{RdW}=$ Rest der Welt, MFI= Monetäre Finanzinstitute (Banken), Versich.= Versicherungen , $\mathrm{AFI}=$ Andere Finanzintermediäre

Quelle: Berechnungen des Autors.

WSI MitTELUNGEN

\begin{tabular}{ll}
\hline \multicolumn{2}{l}{ Übersicht 1: Datenquellen pro Land } \\
\hline Land & Quelle \\
\hline Deutschland & $\begin{array}{l}\text { Deutsche Bundesbank } \\
\text { http://www.bundesbank.de/statistik/statistik_zeitreihen.en.php?open=wirtschaftsdaten }\end{array}$ \\
\hline Frankreich & $\begin{array}{l}\text { Banque de France } \\
\text { http://www.banque-france.fr/fr/stat_conjoncture/series/cptsnatfinann/html/ } \\
\text { cptsnatfinann.htm }\end{array}$ \\
\hline England & $\begin{array}{l}\text { national STATISTICS } \\
\text { http://www.statistics.gov.uk/statbase/TSDtables1.asp }\end{array}$ \\
\hline Spanien & $\begin{array}{l}\text { Banco de Espana } \\
\text { http://www.bde.es/estadis/ccffe/cfcap2e.htm }\end{array}$ \\
\hline Italien & $\begin{array}{l}\text { Banca d'Italia } \\
\text { http://bip.bancaditalia.it/4972unix/homebipentry.htm?dadove=corr\&lang=eng }\end{array}$ \\
\hline Amerika & $\begin{array}{l}\text { Federal Reserve } \\
\text { http://www.federalreserve.gov/releases/z1/Current/accessible/default.htm } \\
\\
\text { Bureau of Economic Analysis } \\
\text { http:///www.bea.gov/national/nipaweb/Ni_FedBeaSna/Index.asp } \\
\text { World Federation of Exchanges } \\
\text { http://www.world-exchanges.org/statistics/time-series/market-capitalization } \\
\text { OECD } \\
\text { http://titania.sourceoecd.org/vl=442651/cl=79/nw=1/rpsv/home.htm }\end{array}$ \\
\hline
\end{tabular}

Quelle: Zusammenstellung des Autors.

WSI MITTEILUNGEN

während die ersten vier Kategorien den nicht-finanziellen Sektor darstellen. Tabelle 1 gibt eine exemplarische Übersicht eines Sektorenmodells für die deutsche Wirtschaft im Jahr 2008.

Die Zeilen der Tabelle 1 repräsentieren das Investitionsportfolio (Vermögen) eines Sektors, während die beiden Längsspalten je Sektor den Finanzierungsmix (Schulden) abbilden. Vermögen und Schulden werden dabei in zwei Arten unterschieden: verbriefte und nicht-verbriefte Instrumente. Unter Verbriefung versteht man die Transformation von illiquiden Instrumenten, wie z. B. Krediten, in am Kapitalmarkt handelbare Instrumente, wie z. B. Schuldverschreibungen (Breidenbach 2005). Weitere Beispiele für verbriefte Instrumente sind gelistete Aktien, Derivate und Fondsanteile.

Durch die von Schmidt et al. (1999, S. 8ff.) entwickelte Technik zur Berechnung von Intermediations- (IK) und Verbriefungskennzahlen (VK) kann man die
Verflechtungen zwischen den Sektoren untersuchen. IK eröffnen dabei einen sektoralen Blick, indem sie darstellen, welcher Anteil von Vermögen/Schulden der nichtfinanziellen Sektoren durch den finanziellen Sektor oder dessen Untersektoren geleitet wird. Grundsätzlich gilt, je höher die IK desto geringer ist die Marktorientierung einer Volkswirtschaft. VK geben Auskunft über den Anteil von Vermögen/ Schulden, der in verbriefter Form gehalten/geschuldet wird. Je höher der Anteil an verbrieften Instrumenten, desto höher ist erwartungsgemäß die Marktorientierung.

Ausgehend von dem Sektorenmodell kann man IK und VK für die jeweiligen Sektoren berechnen. Dementsprechend kalkuliert sich die Vermögens-IK für den nicht-finanziellen Sektor, indem man in Tabelle 1 die Spalten 5a-7b der Zeilen 1-4 addiert und durch die Summe der Zeilen 1-4 teilt. Für 2008 ergibt sich eine Kennzahl von $68 \%$ für Deutschland. Um die Schulden-IK des Nicht-finanziellen Sektors zu erhalten, teilt man die Summe der Spalten 1a-4b der Zeilen 5-7 durch die Summe der Zeilen 5-7. ${ }^{2}$ Für Deutschland ergibt sich 2008 wiederum eine Kennzahl von $68 \%$. Da der finanzielle Sektor in seiner Funktion als Intermediär als ausgeglichen gilt, müssen Vermögens- und SchuldenIK zwangsweise nahezu gleich sein. Die Kennziffern geben an, dass $68 \%$ der Vermögen/Schulden durch den Finanzsektor kanalisiert werden, während nur $32 \%$ eine direkte Verflechtung der nicht-finanziellen Sektoren darstellen, wie z. B. in Form von Aktien des nicht-finanziellen Unternehmenssektors, die von den Haushalten gehalten werden. VK können analog anhand des Sektorenmodels berechnet werden. Die Vermögens-VK des nicht-finanziellen Sektors berechnet sich z. B. als Summe der Spalten 1a, 2a, 3a, 4a, 5a, 6a und 7a der Zeilen 1-4 geteilt durch die Summe der Zeilen 1-4. Es ergibt sich eine Kennziffer von 32 \% für Deutschland im Jahr 2008.

Abgesehen von Kennzahlen für übergreifende Sektoren können diese auch für Untersektoren berechnet werden. So kann man die Intermediation des Finanzsektors auf Banken, Versicherungen und andere Finanzintermediäre unterteilen und auch die Untersektoren des nicht-finanziellen Sektors einzeln analysieren. Wichtige Kennzahlen sind insbesondere die Vermögens-IK von Haushalten, da dieser Sektor klassisch ein Überschusssektor ist, und die Schulden-IK von nicht-finanziellen Unternehmen, da dieser Sektor in der Regel ein Defizitsektor ist, der kontinuierlich neue Finanzierung für Investitionen benötigt. Weiterhin kann die Verflechtung inner-

2 Eine grafische Darstellung der Berechnung kann beim Autor angefragt werden. 
halb des Finanzsektors durch partielle IK und VK untersucht werden, wie z. B. Vermögens-IK von Banken durch Versicherungen oder andere Finanzintermediäre.

\section{3 \\ Entwicklungen der Wirtschafts- und Finanz- systeme}

Im Folgenden werden die Ergebnisse der Analyse (vgl. Fußnote 1) aufgezeigt. Dabei werden wichtige Veränderungen innerhalb des Finanzsektors und speziell innerhalb der Bankenindustrie in den Vordergrund gestellt.

\subsection{VERÄNDERUNGEN IN DEN FUNKTIONEN DER BANKEN}

In einem ersten Schritt geht es um die Rolle und Bedeutung der Banken in der jeweiligen Volkswirtschaft. Ein wichtiges Charakteristikum dabei ist die Funktion der Liquiditätsintermediation. Darunter versteht man den Anteil, den Banken an der Intermediation von Vermögen und Schulden der nicht-finanziellen Sektoren haben und damit an der Bereitstellung der volkswirtschaftlichen Überschüsse für Investitionen. Die Abbildungen 1 und 2 stellen exemplarisch die Vermögens-IK von Haushalten mit Banken und die SchuldenIK von nicht-finanziellen Unternehmen mit Banken dar.

Die Ausgangssituation ist in den sechs Volkswirtschaften unterschiedlich, wobei die eher bank-basierten kontinentaleuropäischen Volkswirtschaften einen deutlich höheren Anteil an Bankintermediation ausweisen. Das deutsche Hausbankensystem erkennt man an der im Vergleich mit den anderen Ländern höchsten Schulden-IK von NFU mit Banken. Abbildung 1 und 2 geben Aufschluss darüber, welcher Anteil des Vermögens von HH bei Banken angelegt wird bzw. welcher Anteil der Gesamtfinanzierung von NFU von Banken getragen wird. Je höher der Anteil, desto mehr Liquidität stellen Banken der Volkswirtschaft zur Verfügung und erfüllen damit ihre Aufgabe, das Geld der Sparer in Investitionsprojekte von Unternehmen zu allokieren. Über den Betrachtungszeitraum sieht man, dass in Deutschland die Vernetzung der Banken mit den beiden anderen Sektoren nur ganz

Abb. 1: Vermögensintermediationskennzahlen von $\mathrm{HH}^{*}$ mit $\mathrm{MFI}^{*}$ - in \% -

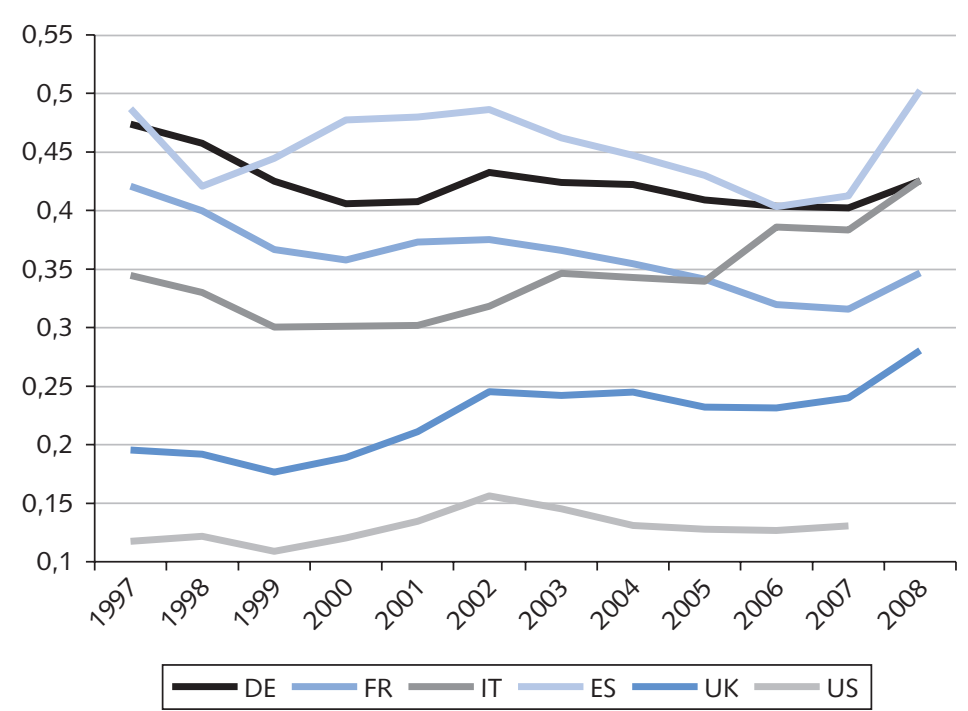

${ }^{*} \mathrm{HH}=$ Haushalte; $\mathrm{MFI}=$ Monetäre Finanzinstitute. Quelle: Darstellung des Autors.

WSI MITTEILUNGEN

Abb. 2: Schuldenintermediationskennzahlen von NFU* mit MFI* - in \% -

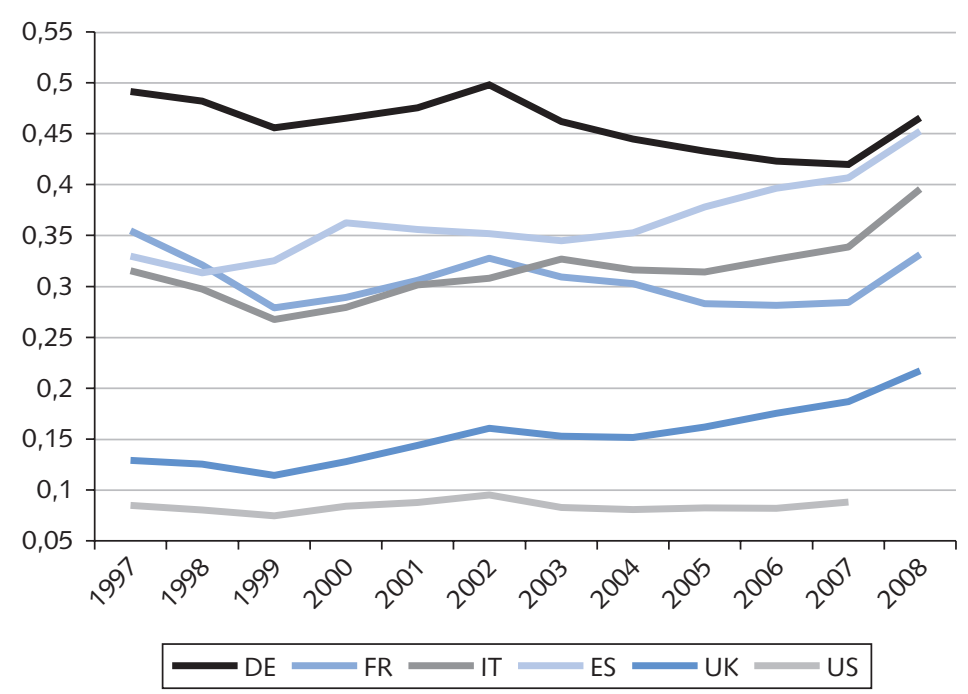

*NFU = nicht-finanzielle Unternehmen; MFI = Monetäre Finanzinstitute. Quelle: Darstellung des Autors.

WSI MITTEILUNGEN

leicht abnimmt, während in Frankreich bis 2007 ein deutlicher Rückgang zu erkennen ist. Im Gegensatz dazu nimmt die Vernetzung in dem markt-basierten englischen System zu. Folglich zeichnet sich die erwartete Abnahme der Dominanz der Banken in Kontinentaleuropa lediglich in Frankreich ab, wohingegen Banken in den anderen Volkswirtschaften ihre Bedeutung sogar teilweise erhöhen können. Besonders auffällig ist die zunehmende Bedeutung englischer Banken, die sich klar von den geringen amerikanischen IK entfernen. Dies ist ein Anzeichen dafür, dass in dem markt-basierten System die Banken teilweise Funktionen vom Markt übernehmen bzw. das System sich zu einer hybriden Form aus Bank- und Marktsteuerung entwickelt.

Neben der Intermediation von Liquidität kommt den Banken durch ihre Beziehungen zu den Schuldnern (Kredite) eine Kontrollfunktion zu. Wegen des Interesses an Zinszahlungen und Tilgungen überwachen Banken die Unternehmensführung z. B. mit Blick auf die Risikobereitschaft. Diese Funktion nimmt ab, wenn die Anreize einer kontinuierlichen und 
Abb. 3: Verbriefungskennzahlen von $\mathbf{M F I}^{*}-$ in $\%-$

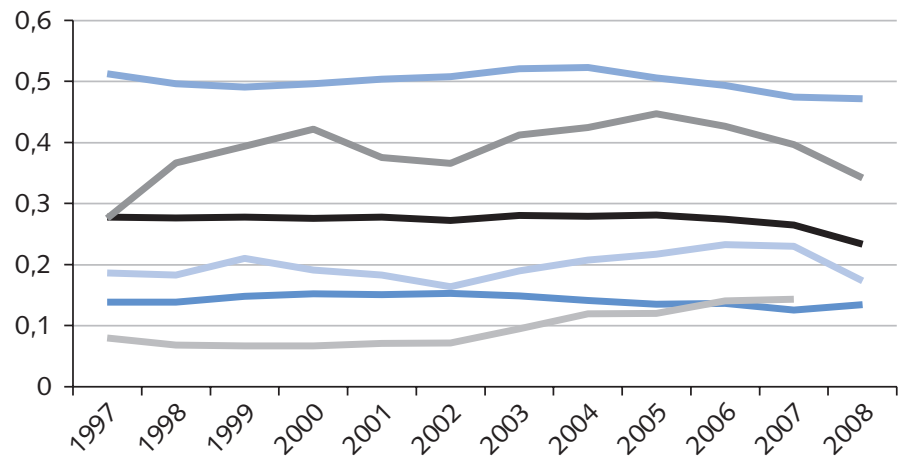

DE $=\mathrm{FR}=\mathrm{IT}=\mathrm{ES}=\mathrm{UK}$

\section{Abb. 4: Nicht vernetzte Intermediationskanäle}

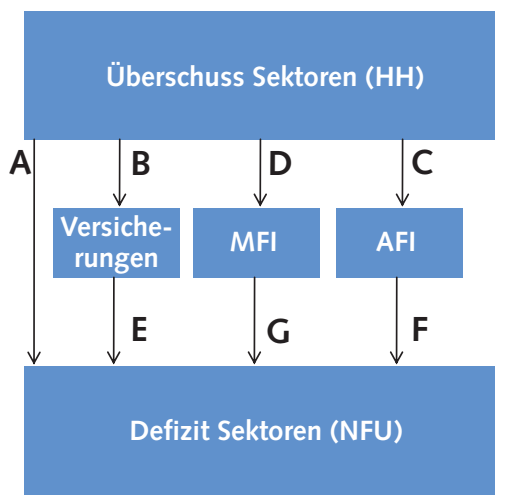

$\mathrm{HH}=$ Haushalte; $\mathrm{NFU}=$ nicht-finanzielle Unternehmen; $\mathrm{MFI}=$ Monetäre Finanzinstitute; $\mathrm{AFI}=$ Andere Finanzintermediäre.

Quelle: Darstellung des Autors.

WSI MITTEILUNGEN

detaillierten Kontrolle verschwinden, da Banken weniger Vermögensgegenstände in Form von Krediten, sondern eher in Form von Anleihen und Aktien halten. Weil diese Instrumente, einen liquiden Markt vorausgesetzt, kurzfristig veräußert werden können, haben Banken kaum Anreize, die zeit- und kostenintensive Aufgabe der Kontrolle zu übernehmen. Folglich zeigt Abbildung 3 die Entwicklung dieser Funktion in den jeweiligen Volkswirtschaften auf. Grundsätzlich gilt, je höher die Verbriefungskennzahlen $(\mathrm{VK})$, desto geringer ist die Kontrollfunktion.

Europaweit werden deutliche Unterschiede in der Kontrollfunktion von Banken erkennbar. Während in Deutschland und England Banken kaum verbriefte Instrumente halten, ist deren Bedeutung in

\section{Abb. 5: Zunahme der Vernetzung des Finanzsektors}

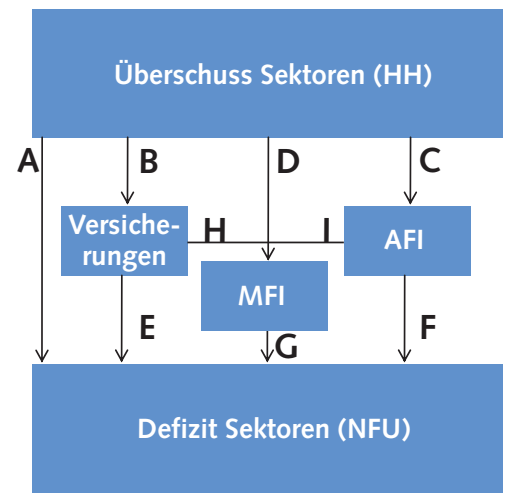

$\mathrm{HH}=$ Haushalte; NFU = nicht-finanzielle Unternehmen; $\mathrm{MFI}=$ Monetäre Finanzinstitute; $\mathrm{AFI}=$ Andere Finanzin termediäre.

Quelle: Darstellung des Autors.

WSI MiTTEILUNGeN

Frankreich stark ausgeprägt. Dass französische Banken sogar einen höheren Anteil an verbrieften Instrumenten als amerikanische Institute halten, ist ein klares Anzeichen dafür, dass die Kontrollfunktion eher durch den Markt ausgeübt werden muss und Frankreich sich auf dem Weg zu einer Transformation seines Wirtschaftssystems hin zu einer Marktorientierung befindet. Trotz der geringen VK in Deutschland ist eine Tendenz zu steigenden Raten zu erkennen, die die Kontrollfunktion abschwächen kann. Mit Ausnahme von Frankreich nähern sich die europäischen Raten über den Betrachtungszeitraum an.

Als Zwischenfazit kann man festhalten, dass ein Bedeutungsverlust des Bankensektors bezogen auf die Ausübung seiner vorteilhaften Funktionen für die
Volkswirtschaft sehr klar in Frankreich zu erkennen ist, während deutsche monetäre Finanzinstitute (MFI) nur leicht an Einfluss verlieren. Im Gegensatz dazu erhöht sich der Einfluss englischer Banken für die Volkswirtschaft und schafft dadurch ein hybrides System aus Markt- und Bankensteuerung.

\subsection{VERÄNDERUNGEN IN DER VERNETZUNG DES FINANZSEKTORS}

Durch Intermediationskennzahlen (IK) einzelner finanzieller Sektoren kann die Verflechtung des Finanzsektors untersucht werden. Je höher die Vernetzung, desto spezialisierter ist der Finanzsektor und folglich kommt marktorientierten Intermediären eine höhere Bedeutung zu. Abbildung 4 zeigt die Intermediation von Überschüssen (Sparen) und Defiziten (Investitionen) über die möglichen Kanäle ohne Vernetzung des Finanzsektors.

Vermögen und Schulden der nichtfinanziellen Sektoren können demnach entweder direkt über den Markt (Kanal A) oder einen Finanzintermediär kanalisiert werden. In einem bankorientierten System, wie z. B. Deutschland, kommt dem Kanal D-G die größte Bedeutung zu. Untersucht wurde, ob Entwicklungen zu mehr Verflechtung festzustellen sind. Ein zentrales Ergebnis fasst Abbildung 5 zusammen.

Mit Ausnahme von Italien zeigt sich eine klare Entwicklung dieser Integrationsform für alle europäischen Volkswirtschaften. Die zunehmende Integration führt dazu, dass der Intermediationskanal D, die direkte Verbindung zwischen Überschusssektoren und den Banken, an Bedeutung verliert. Demnach werden Banken in ihrer Rolle als Vermögensakkumulator durch nicht-MFI ersetzt, wobei sie ihre Finanzierung durch die Kanäle $\mathrm{H}$ und I erhalten. Dennoch behalten Banken ihre dominante Rolle in der Finanzierung von Defizit Sektoren (G>A; G>E; G>F). Folglich üben Banken weiterhin die Kontrollfunktion von Unternehmen aus, mit oben beschriebenen Einschränkungen, wobei sie in ihrer Funktion als Liquiditätsvermittler auf nicht-MFI angewiesen sind. Die zunehmende Bedeutung von Versicherungen und anderen Finanzintermediären (AFI) als Vermögensakkumulatoren führt zu einer Abnahme der Verbreitung klassischer Bankeinlagen hin zu komplexeren Produkten wie z.B. Investmentfondsanteilen. 
rar

\section{Fazit}

Die grundlegenden Ergebnisse der Studie sprechen für eine veränderte Rolle von Banken, sowohl in bank- wie auch marktbasierten Systemen. Während in England monetäre Finanzinstitute (MFI) an Bedeutung gewinnen und Funktionen des Marktes übernehmen können, zeigen sich in Deutschland leichte und in Frankreich sehr starke Tendenzen zu einer höheren Marktorientierung. Damit entsteht eine neue, hybride Form von Wirtschaftssystemen, die eine starke Bankenindustrie mit nicht-MFI vernetzt, womit teilweise Bankfunktionen durch den Markt übernommen werden. Banken scheinen sich in diesem neuen System zunehmend zu spezialisieren und teilweise Funktionen an nicht-MFI abzugeben. Ob diese Entwicklung durch die Banken getrieben wird oder sie sich den ändernden Rahmenbedingungen anpassen müssen, kann aus der Studie nicht abgeleitet werden. Dennoch wird deutlich, dass die ehemaligen klaren Grenzen zwischen bank- und marktbasierten Volkswirtschaften verschwim- men. Demnach ist eine internationale Konvergenz von Wirtschaftssystemen zu erkennen. Diese Ergebnisse stimmen mit einer aktuellen Analyse der Europäischen Zentralbank (2009) überein, die eine Annäherung von Finanzsystemen belegt. Da Ansichten über die Vorteilhaftigkeit des neuen, hybriden Systems stark widersprüchlich sind (Deidda/Fattouh 2008; Boot/Thakor 2009), wäre es von besonderem Interesse, diese näher zu analysieren und Aussagen über die Stabilität des Wirtschaftssystems und die Konsequenzen auf das Wachstumspotenzial der Volkswirtschaften abzuleiten.

\section{LITERATUR}

Allen, F./Gale, D. (1995): A welfare comparison of intermediaries and financial markets in Germany and the US, in: European Economic Review 39, S. 179-209

Allen, F./Santomero, A.M. (2001): What do financial intermediaries do?, in: Journal of Banking and Finance 25, S. 271-294

Boot, A./Thakor, A.V. (2009): The Accelerating Integration of Banks and Markets and its Implications for Regulation, Manuskript, The Oxford Handbook of Banking

Breidenbach, M. (Hrsg.) (2005): Real estate securitisation - Assetbacked security financing for the property industry, Köln

Deidda, L.G./Fattouh, B. (2008): Bank, Financial Markets and Growth, Journal of Financial Intermediation 1, S. 6-36
Europäische Zentralbank (2009): The External Financing of Households and Non-financial corporations: A Comparison of the Euro Area and the United States, in: Monthly Bulletin, April, S. 69-84

Levine, R. (2002): Bank-Based or Market-Based Financial Systems:

Which is Better?, Manuskript, University of Minnesota

Miller, G. (1997): The Obsolescence of Commercial Banking, in: Journal of Economic Literature 35, S. 1012-1037

Schmidt, R.H./Hackethal, A./Tyrell, M. (1999): Disintermediation and the Role of Banks in Europe: An International Comparison, in: Journal of Financial Intermediation 8, S. 36-67

Tadassee, S. (2000): Financial Architecture and Economic Performance: International Evidence, Manuskript, University of South Carolina 